Ophthalmologica 1944;107:50-51

\title{
A propos de la dacryo-cysto-rhinostomie
}

\begin{tabular}{|l|l|l|}
\hline M. & Marc & Amsler \\
\hline
\end{tabular}

Travail de la Clinique ophtalmologique de I'Université de Lausanne [Dir.: Prof. M. Amsler]

Nous faisons, a la Clinique de Lausanne, une moyenne de 10 dacryo-cysto-rhinostomies plastiques par an. Cette operation fonctionnelle a complètement supplanté, depuis dix ans,

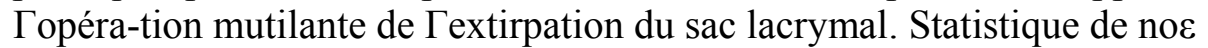

Bangerter, Beil·räge zur Diagnostik und Therapie des Tränens. 51

60 derniers opérés : 34 femmes, 26 homines; age : de 9 à 29 ans : 11 malades; de 30 à 59 ans :

37 malades ; de 60 à 74 ans : 12 ma-lades. Technique : anesthésie parfaite ; éclairage «

scialytique »; grande fenêtre osseuse ; sutures proí'ondes à la soie. Pas d'inci-sions

canaliculaires préalables, ni de sondages répétés! L'indica-tion de Гopération de Toti-BourguetDupuy-Dutemps est donnée par la dacryo-cystite purulente chronique, même fistulée. Plus le sac est dilate, plus Гintervention est facile. Les résultats sont excellents dans Гimmense majorité des cas. Les très rares échecs sont constates immédiatement ; les « rechutes » tardives ne sont pas a craindre.

(Aus der Universitäts-Aug < enklinik Bern [Direktor: Prof. Goldmann].)

Beiträge zur Diagnostik und Therapie des Tränens.

Von A. BANGERTER.

Zeigt die negative Fluoresceinprobe, daß es sich um eine Tränenabflußstörung handelt, ist es unsere Aufgabe, Art und Sitz des Hindernisses festzustellen, um eine entsprechende Therapie durchführen zu können. An Hand des nachfolgenden Schemas maclien wir einen Vorschlag zur systematischen Untersuchung der Tränenabflußwege und Indikationsstellung für die Therapie.

I. Spülung ohne Vorschieben der Kanüle:

1. positiv: Tränenpunkt zeigt: a) abnorme Stellung (eversio):Myrtenblatt, b) abnorme Lage: Punktplastik, c) Stenose:Dehnung, evtl. Punktplastik;

2. negativ: siehe II.

II. Vorschieben der Kanüle: 1. unmöglich,

2. möglich,

a) Spülung ++: Canaliculusstenose bzw. Ven-til: Elektrokoagulation und Dauersonde; Spülung -

: Kanalstenose siehe III.

III. Privin in den Kanal (nur bei frischen Stenosen), 